\title{
Line-profile variability in the Wolf-Rayet stars WR 135 and WR 111
}

\author{
L. Koesterke ${ }^{1, \star}$, W.-R. Hamann ${ }^{1}$, and T. Urrutia ${ }^{1,2}$ \\ 1 Professur Astrophysik, Universität Potsdam, Am Neuen Palais 10, 14469 Potsdam, Germany \\ 2 Astrophysikalisches Institut Potsdam, 14482 Potsdam, Germany
}

Received 27 June 2001 / Accepted 7 September 2001

\begin{abstract}
We have obtained time-resolved observations of line-profile variations of the two Wolf-Rayet stars WR 135 and WR 111. The spectra, taken during two consecutive nights, cover a broad range from 4470 to $6590 \AA$. The profile variability of the C III emission line at $5696 \AA$ in WR 135 is shown in detail. The principal difficulties to constrain the velocity law from the frequency drift of discrete spectral features is discussed, emphasizing the crucial dependence on the adopted location of the line-emission region, and the possible necessity to distinguish between the motion of structures and the flow of the matter. - Full access to the observational data is provided via anonymous file transfer.
\end{abstract}

Key words. stars: mass-loss - stars: Wolf-Rayet

\section{Introduction}

Wolf-Rayet (WR) stars are highly evolved objects which show products of nuclear hydrogen and helium burning at the surface. Their spectra, which are formed in a dense and fast stellar wind, are dominated by broad emission lines of either helium and nitrogen (WN-type) or helium, carbon and oxygen (WC-type). In recent years it became clear from various observations (see Brown et al. 1995 for an overview) that the winds are not homogeneous but structured, while the so-called standard model for WR stars is based on the simplifying assumptions of spherical symmetry, homogeneity and stationarity. The most obvious indications of wind structures are narrow emission features superposed on the emission lines which persist on timescales of about 10 hours and which drift generally from the center towards the edge of the lines. These features are assumed to arise from regions of enhanced emission (clumps, blobs) which are accelerated in radial direction.

In a series of papers, Lépine and co-workers have presented both mathematical tools to investigate line profile variations (LPVs) (Lépine et al. 1996) and time-resolved observations of various WR stars (Lépine \& Moffat 1999; Lépine et al. 1999, 2000). They conclude from a wavelet analysis that very many $\left(\approx 10^{4}\right)$ discrete wind emission

Send offprint requests to: L. Koesterke,

e-mail: lars@astro.physik.uni-potsdam.de

* Visiting astronomer, German-Spanish Astronomical Center, Calar Alto, operated by the Max-Planck-Institut für Astronomie, Heidelberg, jointly with the Spanish National Commission for Astronomy. elements (DWEEs) contribute simultaneously to the spectral lines. In terms of the usual $\beta$-law for the velocity field they find low radial accelerations, i.e. substantially higher $\beta$ values compared to the canonic value of $\beta=1$ (Lépine et al. 1999). In their most recent paper (Lépine et al. 2000) high-quality observations of WR 135 are presented and analyzed. Stochastic and structured patterns at the $2 \%$ level are found in the C III line at $5696 \AA$, and low radial accelerations are derived from comparison with simple clump models.

In the present paper we introduce our own, timeresolved observations of the two galactic WR stars WR 135 (WC 8) and WR 111 (WC 5). The parameters of both program stars have been determined before from non-LTE spectral analyses by Koesterke \& Hamann (1995). For WR 135 the results were confirmed recently by Dessart et al. (2000).

In Sect. 2 observations and data reduction are described, while only the LPVs in the C III line at $5696 \AA$ of WR 135 are presented in detail (Sect. 3). Section 4 discusses the interpretation of observed wavelength drifts. A summary is given in Sect. 5 .

\section{Observation and data reduction}

The observations were carried out by one of us (L.K.) on July 20-22, 1999, with the Calar Alto $3.5 \mathrm{~m}$ telescope. Using the TwIN spectrograph, two spectral regions (blue: 4470 to $5500 \AA$ red: 5500 to $6590 \AA$ ) were covered simultaneously with a spectral resolution of about $1.1 \AA$. The data were reduced with IDL in the usual way. After 
Table 1. Overview on the observations.

\begin{tabular}{lllrr}
\hline $\begin{array}{l}\text { Date } \\
1999\end{array}$ & Star & $\begin{array}{l}\text { Start JD } \\
-2451380\end{array}$ & $\begin{array}{r}\text { Duration } \\
\text { [min] }\end{array}$ & $\begin{array}{r}\text { No. of } \\
\text { spectra }\end{array}$ \\
\hline Jul. 20/21 & WR 135 & 0.39167 & 30 & 6 \\
Jul. 20/21 & WR 111 & 0.41736 & 13 & 5 \\
Jul. 20/21 & WR 111 & 0.45556 & 7 & 4 \\
Jul. 20/21 & WR 135 & 0.46250 & 6 & 4 \\
Jul. 20/21 & WR 135 & 0.56111 & 87 & 52 \\
\hline Jul. 21/22 & WR 111 & 1.38659 & 124 & 70 \\
Jul. 21/22 & WR 135 & 1.47292 & $254^{*}$ & 145 \\
\hline
\end{tabular}

*Bad weather from 130th to 150 th min.

flat and bias correction the spectra have been extracted, corrected for the background, and wavelength calibrated. The $S / N$ ratio in the continuum is 60 , typically, but differs between the individual exposures due to unstable weather conditions. Note that the spectral region around $5500 \AA$ is corrupted by the beam splitter. An overview on the observations is given in Table 1 .

\section{Variation of the C III line profile}

The C III line at $5696 \AA$ is known to show the most pronounced profile variations. We focus on our observations of WR 135 in the night July 21/22, 1999, because this is the longest of our time series. In order to uncover the variations we proceed in the following way: (a) all spectra are normalized with respect to a straight continuum which is fixed at two points, bluewards (5640 $\AA$ ) and redwards (5750 $\AA$ ) of the C III line; (b) a template profile is subtracted from each individual observation.

For the template we take the 95-percentile of all our spectra from that star over both observing nights. (The $n$-percentile at each wavelength point has by definition a value which is higher than $n \%$ of the individual measurements, but lower than the rest). In Fig. 1 we show this template spectrum together with a median and a minimum profile which are obtained as 50-percentile and 5-percentile, respectively. With the 95-percentile template the LPVs become visible best, but our discussion does not depend on this particular choice. As can be seen, the flux in the top of the line profile varies by about \pm 0.14 , in units of the continuum flux (which is $\pm 2 \%$ relative to the line top flux). As the percentiles are a kind of averages over about 200 spectra (in fact, the 50 -percentile is almost identical to the arithmetic mean), their statistical noise is very small with an $S / N$ ratio better than 1000 .

In Fig. 2, the variation of the $\mathrm{C}$ III line profile is shown in a grey-scale representation. Note that the time at the right-hand ordinate is converted at the left-hand axis in units of flight times $t_{\text {flight }}=R_{*} / v_{\infty}$. With a stellar radius of $R_{*}=2.6 R_{\odot}$ and the terminal velocity $v_{\infty}=1300 \mathrm{~km} \mathrm{~s}^{-1}$ (from Koesterke \& Hamann 1995, in fair agreement with Dessart et al. 2000) one obtains $t_{\text {flight }}=23.2 \mathrm{~min}$. The wavelength scale (top abscissa) is translated into Doppler shift velocities relative to the line

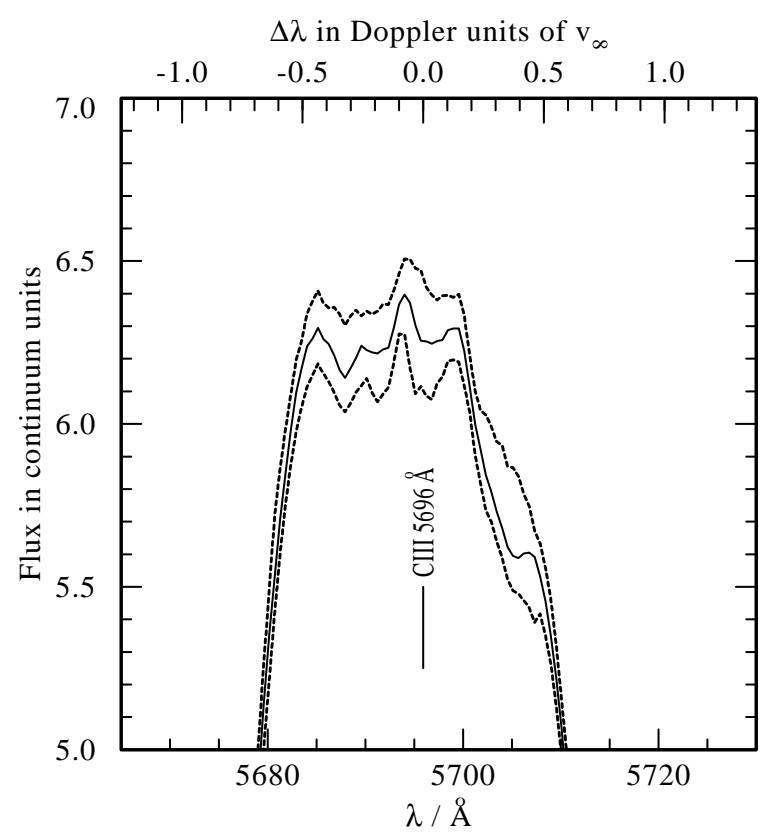

Fig. 1. Top of the C III line profile at $5696 \AA$ from WR135 (full line), obtained as median value of all our spectra from that star (both observing nights). The dashed curves give a minimum and a maximum profile, calculated as the 5-percentile and 95-percentile at each wavelength point.

center (bottom abscissa). The difference to the mean template shows a couple of features which are drifting away from the line center. It is not clear if one should consider the bright features as excess of emission, or the dark structures as lack of emission (compare Figs. 2 and 4). However the emission of that line is dominated by recombination processes, so one should not expect the dark features to be absorption - in contrast to the "discrete absorption components" observed in the UV resonance lines of O stars.

The observations of WR 111 are processed in the same way as those of WR 135 . The profile variations in the C III $5696 \AA$ line are less pronounced in WR 111, compared to WR 135 (cf. Lépine \& Moffat 1999), and at the top level of that line profile (which has only a peak height of about 2.0 in continuum units) the $S / N$ ratio of our spectra is not sufficient to make them clearly visible. Moreover, the time longest series for WR 111 covers only two hours. Hence these data are probably less useful and we do not present them here, but nevertheless they are provided via our ftp site.

\section{On the interpretation of the wavelength drift}

The drift in wavelength contains information about the wind dynamics. Lépine \& Moffat (1999) applied sophisticated methods on similar (and better) data, concluding that the velocity law in WR 135 and other WR stars is less steep than commonly assumed. We will not repeat this detailed analysis, but use our present data and some simple kinematic modeling to discuss some general aspects of the information which is contained in those spectra. 


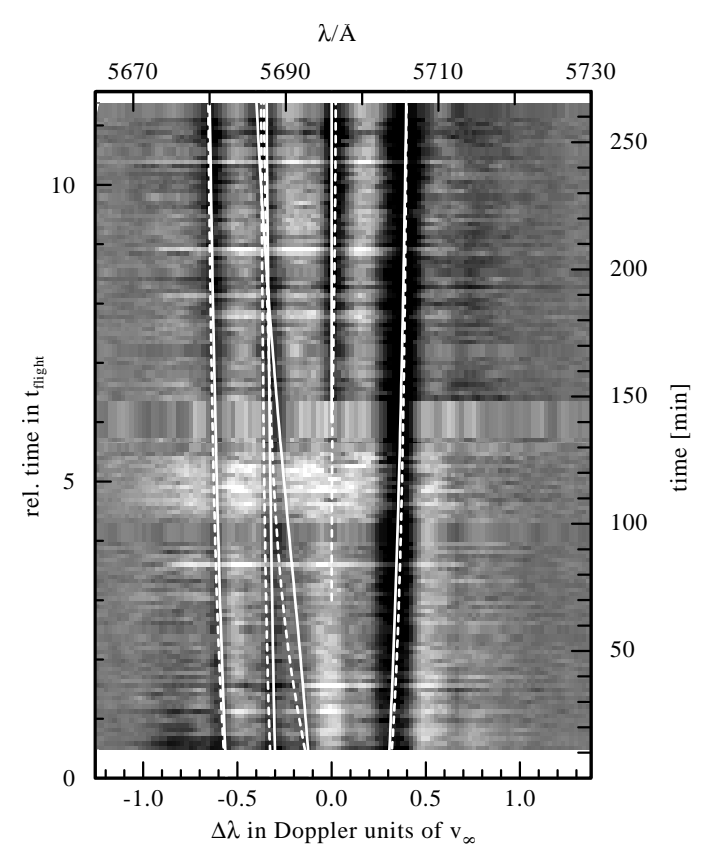

Fig. 2. LPVs in the CIII line at $5696 \AA$ of WR 135 on July $21 / 22,1999$. A mean template has been subtracted. The $y$-axis is given in units of $t_{\text {flight }}=R_{*} / v_{\infty}$. Slowly outwards moving patterns darker or brighter than the template (black and white, respectively) are visible. Superimposed are kinematic curves fitted tentatively to dark features, assuming the usual $\beta$-law with $\beta=4$ (solid) and $\beta=1$ (dashed), without restricting the line-emitting region.

In a straight-forward interpretation, one might assume that the drifting features are originating from some density structures (clumps or voids). If these features are fully advected with the stellar wind, their wavelength drift reflects the velocity law $v(r)$. The time to reach an outflow velocity $v$ is obtained by integrating

$t=\int \frac{\mathrm{d} r}{v(r)}$

An integration constant $t_{0}$ is a free parameter for each individual feature and reflects the different time when the corresponding structure is passing some reference radius $r_{0}>R_{*}$. The observed wavelength shift is $\Delta \lambda=\mu v(t) / c$. Therefore, a second free parameter is the projection factor $\mu$, i.e. the cosine of the angle between the direction to the observer and the direction in which the structure is flying off from the star. In contrast to the "discrete absorption components" in UV resonance lines, which are formed between the stellar disk and the observer, $\mu$ can have any values between -1 and +1 for wind emission elements.

In Fig. 2 we have roughly fitted some $\Delta \lambda(t)$ to the dark structures in the dynamical spectrum by adjusting $t_{0}$ and $\mu$ "by eye". This turned out to be easy since the parameter $\mu$ is almost fully determined by the maximum wavelength shift of each feature. For the velocity law we adopt the usual " $\beta$-law" of the form $v(r)=v_{\infty}\left(1-R_{*} / r\right)^{\beta}$, testing $\beta=1$ (dashed) and $\beta=4$ (solid line). The whole velocity range from (almost) zero to $v_{\infty}$ is plotted, i.e. no restriction is assumed yet for the line-emitting region.

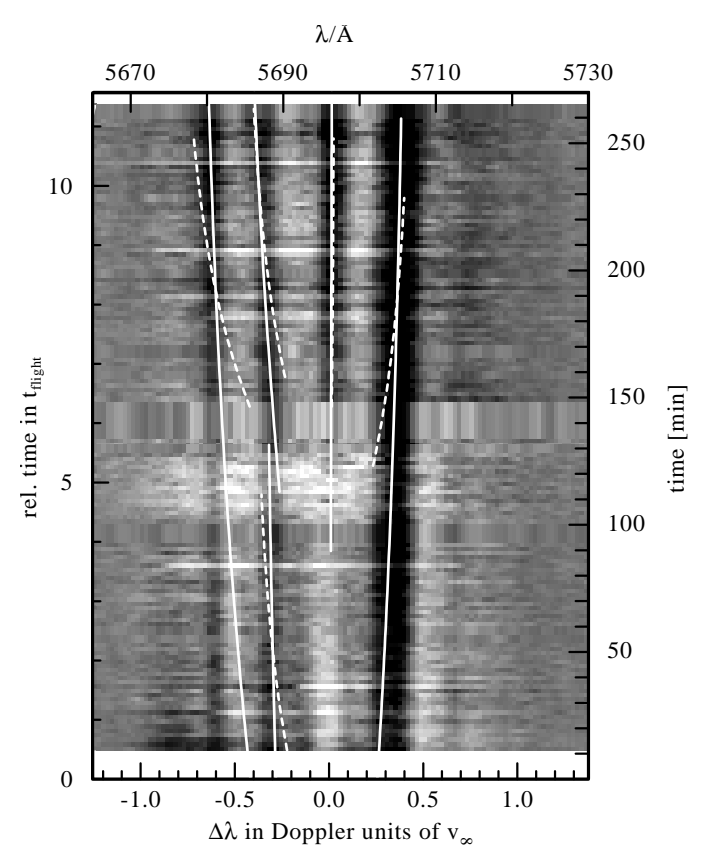

Fig. 3. Same as Fig. 2, but now restricting the kinematic curves to the velocity range of the line-emission region (890 \pm $240 \mathrm{~km} \mathrm{~s}^{-1}$ following Lépine \& Moffat 1999). As can be seen, the $\beta=1$ curves (dashed) now cannot be fitted to the observation, as their velocity drift is much too fast.

As the figure shall demonstrate, the features can be fitted equally well by both, dashed and solid curves, i.e. the velocity law exponent cannot be constrained this way.

An essential conclusion of Lépine \& Moffat (1999) was that the acceleration in WR winds is slow. Their result for WR 135 is $\beta R_{*}>20 R_{\odot}$, which means $\beta>7$ for a plausible stellar radius. Why do the above kinematic fits not exclude a $\beta$ as low as 1 ? The reason is, that we did not restrict yet the region of line formation. The acceleration is decreasing towards large radii, and hence arbitrarily slow wavelength drifts can be reproduced by adjusting the two available free parameters. The essential information needed is thus the range (in velocity or radius) of line formation.

Lépine \& Moffat (1999) derived that range by an inverse method from the (average) line profile, performing a "spherical deprojection" under some simplifying assumptions. For the formation of the CIII line in WR 135 they obtain a velocity range of $890 \pm 240 \mathrm{~km} \mathrm{~s}^{-1}$ (corresponding to a fraction of $0.46 \ldots 0.80$ of their $\left.v_{\infty}=1405 \mathrm{~km} \mathrm{~s}^{-1}\right)$. In Fig. 3 we have restricted the kinematical curves to that range in velocity, and re-adjusted the fit parameters as good as possible. Now it is obvious that the $\beta=1$ curves (dashed) predict much faster wavelength drifts than observed. Moreover, the structures are crossing the line emission region faster than their observed lifetime.

However, under the assumption that the $\mathrm{C}$ III line is formed at velocities higher than about $0.8 v_{\infty}$, i.e. further out than stated by Lépine \& Moffat (1999), even a $\beta$ value as small as unity is compatible with the dynamical spectra 
because the acceleration decreases towards large radii in any $\beta$ law. (Implicitly, this can also be seen from Fig. 14 in Lépine \& Moffat 1999.) This example demonstrates the crucial role of the adopted location of the line-emission region for deriving velocity-law parameters.

Alternatively, this location might be extracted from our line-blanketed non-LTE models for WC atmospheres (cf. Gräfener et al. in prep.). Selecting a model which reproduces the whole WR 135 spectrum reasonably well, the $\mathrm{C}$ III line is found to be formed between 0.78 and $0.95 v_{\infty}$ if the model is calculated with $\beta=1$. As stated above, for this line-emission region the wavelength drift is compatible with a small $\beta$. However, the observed spectrum is also reproduced well by a model with $\beta=4$. In that model, the $\mathrm{C}$ III line-emission region lies at smaller velocities $(0.40$ to $\left.0.59 v_{\infty}\right)$, and for that range the wavelength drift requires a high $\beta$. Thus, for both models (with $\beta=1$ and 4 , respectively) the predicted velocity range of the $\mathrm{C}$ III lineemission region and the observed wavelength drift are internally consistent.

We want to note here that we are not yet fully confident in our models with respect to the C III $5696 \AA$ line, because its formation is corrupted by complicated details of the radiative transfer (dielectronic recombination, stimulated emission).

Lépine et al. (2000) emphasized that each moving feature in the observed LPV represents many individual "wind emission elements", which only by random superposition sometimes form an outstanding signal. However, in optically thin emission as given in the considered $\mathrm{C}$ III line, any superposition should also reflect the wind dynamics in the same way as a single event, despite a possible broadening or narrowing of the feature's shape, so that the above kinematic considerations remain valid at least in first approximation.

As another caveat, we want to emphasize that the interpretation of the LPVs as reflecting directly the matter velocity might not be adequate. The drifting features are formed in local structures of the wind, which cause some excess absorption or emission in the line profile. These structures might be enhancements or rarefactions of the density, which enters quadratically the recombination rates. Disturbances in the velocity gradient can also affect the $\mathrm{C}$ III line emission via the complicated coupling between the non-LTE population numbers.

The frequency drift observed in the LPV reflects the velocity of the matter (via Doppler effect) at the place of the local structure. But only if that structure is fully advected with the flow of matter is the interpretation as straightforward as adopted above. Such full advection is suggested by the over-simplified picture of individual and persistent clouds separated by void space. However, in a radiation-driven wind, structures might actually propagate relatively to the matter flow.

Two corresponding effects are known in line-radiation driven winds. Abbott (1980) discovered a new type of radiative-acoustic waves, and Owocki et al. (1988) reported on reverse shocks due to the so-called de-shadowing

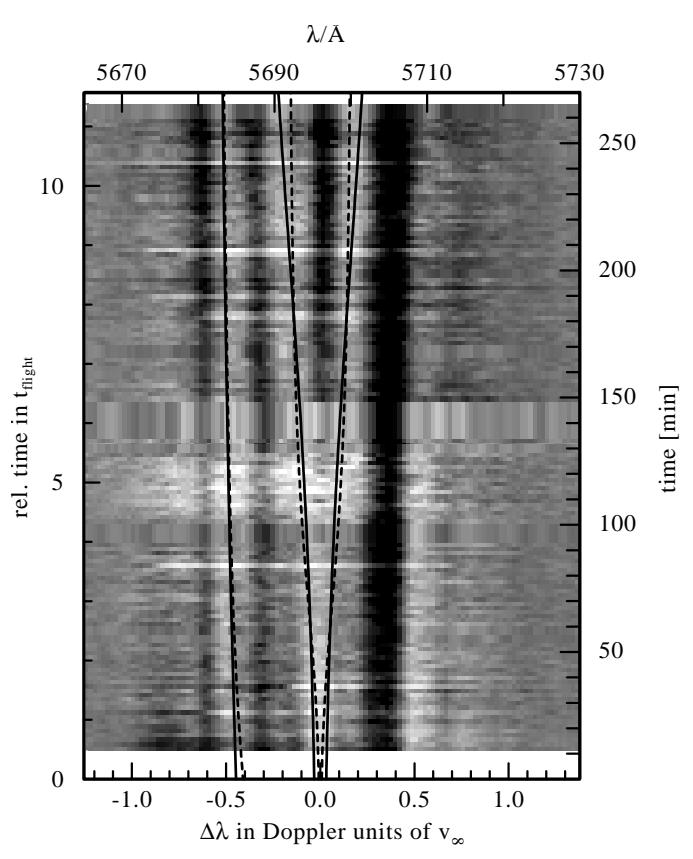

Fig. 4. Same as in Fig. 2 but with tentative fits to bright features (solid: $\beta=4$; dashed: $\beta=1$ ).

instability. Due to these mechanisms, structures can propagate upstream with high velocity. As a result, the structures still move outwards, but slower than the matter flow which is passing through the structures.

Hamann et al. (2001) show that e.g. a structure propagating upstream with $0.4 v_{\infty}$ relative to the matter, while the matter itself streams according to a $\beta=1$ law, leads to a frequency drift very similar to the case of a fully advected pattern moving according to a $\beta=4$ law. The idea of upstreaming patterns is very attractive since it brings the low $\beta$ of about 1 from the theory of radiation driven winds (Pauldrach et al. 1986; Friend \& Abbot 1986) in accordance with the higher $\beta$ values derived by Lépine and coworkers (e.g. Lépine et al. 1999).

\section{Summary}

New time-series observations have been obtained for the two Wolf-Rayet stars WR 135 and WR 111. The profile variability of the C III emission line at $5696 \AA$ in WR 135 is shown in detail. Discrete features, drifting away from the line center, contain information about the wind velocity field. By means of simple kinematic modeling we discuss the possibilities for restricting the velocity-law exponent $\beta$. Adopting the location of the line-emission region as derived by Lépine \& Moffat (1999), $\beta$ must be much larger than unity; otherwise, the variable spectral features should show a faster drift in wavelength. However, this conclusion depends sensitively on the location of the line-emission region. If assuming that the line is formed further out in the wind, low values of $\beta$ cannot be ruled out.

Moreover, one must possibly distinguish between the motion of the structures in the wind which cause the 
drifting spectral features, and the velocity of the matter flow, as the former may propagate relatively to the latter and thus mimic a flatter velocity law.

An advantage of the presented observations is their broad wavelength coverage from $4500 \AA$ to $6500 \AA$. In principle this allows the simultaneous investigation of several spectral lines, e.g. from different ions. According to Lépine et al. (2000) such work could improve our knowledge concerning (1) the size and location of the line-emitting region, (2) the ionization structure, (3) the wind dynamics, and (4) the radial trend of the clumpiness. However, the $S / N$ ratio of our data is possibly not sufficient for studying lines with a low level of variability. Anyhow, such thoroughly study is beyond the scope of our present paper.

Full access to the observational data is provided via anonymous file transfer:

ftp: ftp.astro.physik.uni-potsdam.de

user: anonymous

password: your email address

files: pub/lkoesterke/lpvdata/*

\section{References}

Abbott, D. C. 1980, ApJ, 242, 1183

Brown, J. C., Richardson, L. L., Anthokin, I., et al. 1995, A\&A, 295, 725

Dessart, L., Crowther, P. A., Hillier, D. J., et al. 2000, MNRAS, 315,407

Friend, D. B., \& Abbott, D. C. 1986, ApJ, 311, 701

Gräfener, G., Koesterke, L., \& Hamann, W.-R. 2001, A\&A, in preparation

Hamann, W.-R., Brown, J. C., Feldmeier, A., \& Oskinova, L. M. 2001, A\&A, in press

Koesterke, L., \& Hamann, W.-R. 1995, A\&A, 299, 503

Lépine, S., \& Moffat, A. F. J. 1996, ApJ, 466, 392

Lépine, S., \& Moffat, A. F. J. 1999, ApJ, 514, 909

Lépine, S., Eversberg, T., \& Moffat, A. F. J. 1999, ApJ, 117, 1441

Lépine, S., Moffat, A. F. J., St-Louis, et al. 2000, AJ, 120, 3201

Owocki, S. P., Castor, J. I., \& Rybicki, G. B. 1988, ApJ, 335, 914

Pauldrach, A., Puls, J., \& Kudritzki, R. P. 1986, A\&A, 164, 86 